\title{
ORIGIN OF THE DA AND NON-DA WHITE DWARF STARS
}

\author{
Harry L. Shipman \\ Physics and Astronomy Department, University of Delaware \\ Newark, DE 19716 USA
}

\section{INTRODUCTION}

Understanding the origin of DA and non-DA stars -- the bifurcation of the white dwarf cooling sequence -- is one of oldest and perniciously difficult problems in the white dwarf research field. Processes which could play a role have been discussed for years. For example, the idea of diffusive element separation was suggested by Schatzman (1958). The suggestion of convective mixing goes back at least as far as Strittmatter and Wickramasinghe (1971) and Shipman (1972), and this process was actively discussed by a number of authors in the 1970s (see the excellent resume by D'Antona and Mazzitelli 1979).

Recently, two developments have sharpened the debate and brought this question back to center stage. First has been the appearance of two reasonably detailed theoretical proposals for the origins of the bifurcation of white dwarfs into DA and non-DA stars. A second development has been a considerable improvement in our understanding of the composition of the central stars of planetary nebulae, of the nebulae themselves, and of the conditions under which they are formed. An excellent review of the planetary nebula evidence will soon be available (Torres-Peimbert 1988).

My purpose in this paper is to review various proposals for the bifurcation of the white dwarf cooling sequence. There is only a small overlap with two reviews on the chemical composition of white dwarfs presented in the past year (Shipman 1987, 1988). This review complements Sion (1986), in that I focus more on particular mechanisms and scenarios while sion focuses primarily on the phenomenology. I begin (section II) with a discussion of the evidence in favor of "primordial" theories, theories in which the basic bifurcation of the white dwarf sequence is rooted in events which predate the white dwarf stage of stellar evolution. Section III discusses a competing type of theory, the "mixing" theories in which processes occurring during the white dwarf stage are responsible for the existence of DA or non-DA stars, and the evidence in favor of them. In section IV, the difficulties faced by each class of theory are discussed. Section VI presents a "modest proposal" -.. a scenario which seems to me to be most consistent with the current evidence. 
General Description: This class of theory is one where the distinction between DA and non-DA is hypothesized to lie in the pre-white dwarf stage of stellar evolution (see Figure 1 on the next page). Most recently, the recognition of the thermal pulse phenomenon in the evolution of AGB stars (see, e.g., Renzini 1983, Iben and Renzini 1983, Iben 1984, Iben and MacDonald 1985, 1986, Renzini 1988) has led to a specific proposal that the phase of the onset of the superwind phase determines whether or not the superwind will carry off all of the $H$ envelope. If the $H$ envelope is carried off, a bare He core remains which will turn into a non-DA; if some of the $H$ envelope remains, the remnant will be a $D A$ star with a $H$ shell of order $10^{-4}$ solar masses.

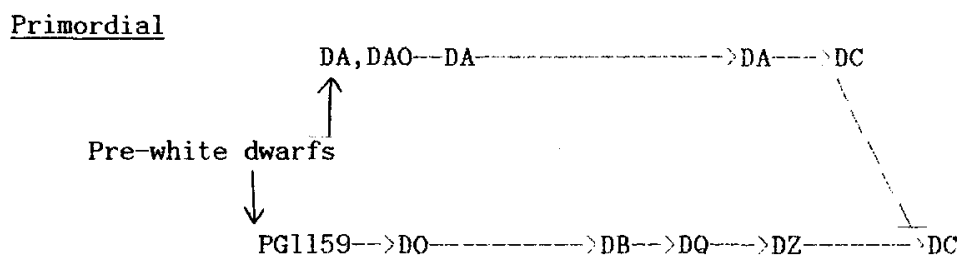

Mixing

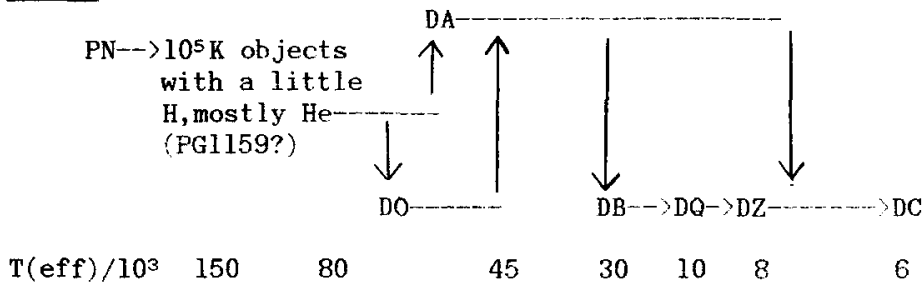

Figure 1: Two extreme explanations for the chemical evolution of white dwarf stars.

Several authors have composed variations on this theoretical theme. For example, Schonberner suggested some years ago (Schonberner 1977) an evolutionary connection between the well-known set of $\mathrm{H}$-deficient red giants (e.g., R Cor Bor stars) and the non-DA stars. Most discussion about H-deficient stars seems to presume that they contribute only a few percent of the remnants of lowmass stars; Schonberner pointed out that, at the time, the deathrate of $\mathrm{H}$-deficient stars was quite uncertain and could be considerably higher. A more modern estimate would be useful.

The Nature of Central Stars of Planetary Nebulae: One strong reason for believing in primordial scenarios for the origin of the white dwarf bifurcation, is that signs of this bifurcation are seen in the central stars of planetary nebulae (CSPN). In the standard classification system for CSPN, two of five. 
classes of central star spectra are ostensibly hydrogen-poor. The O VI stars are defined by the presence of the $O$ VI doublet at 3811-3834 A, and the WC stars are analogous to the main sequence Wolf-Rayet stars. Sion, Liebert, and Starrfield (1986) mentioned the possibility of a connection between the $O V I$ stars and the PG1159 group of white dwarf stars.

Because of the difficulties in determining whether a spectrum with weak $H$ lines in fact indicates a deficiency of $\mathrm{H}$, a most important recent development has been the analysis of the spectra of a number of CSPN by Kudritzki and collaborators (see Mendez et al. 1986, 1988; Mendez 1987, Kudritzki 1988). A significant number of CSPN show a $\mathrm{H}$ deficiency, with an extreme being NGC 246's central star with $\mathrm{T}$ (eff) $=130,000 \mathrm{~K}, \mathrm{H} /$ total $<0.1$, He/total $=0.5-0.9$, and $\mathrm{C} /$ total $=0.1-0.5 .6$ of the 22 CSPN with He abundances in Mendez et al. (1988) have $\mathrm{y}(=\mathrm{N}(\mathrm{He} /[\mathrm{N}(\mathrm{H}) / \mathrm{N}(\mathrm{He})]) ; 0.15$; this is $27 \%$ of the total. At the other end of the distribution are some CSPN which are distinctly He-poor such as the central star of NGC 7293 with $y=0.009$.

Were all of the CSPN as extreme as the two stars described above, the connection between these objects and the two compositional classes of white dwarf stars would be quite clear, especially considering that the proportion of $\mathrm{H}-$ deficient objects among the CSPN and among the white dwarf stars are quite similar. In fact, the situation is somewhat more complicated. Most H-deficient CSPN show much more modest enhancements, and among the others a "normal" (i.e., solar) He/H ratio seems more common than a genuine He deficiency. Nevertheless, the idea of a connection between the H-deficient central stars and at least the hottest non-DA white dwarf stars has been discussed rather extensively within the planetary nebula community, if not within the white dwarf community.

There has been considerable debate in the planetary nebula literature as to whether the H-deficient CSPN have any other distinguishing features. Renzini (1988) argues on theoretical grounds that Pop II stars and massive white dwarfs (descended from massive progenitors) should have a greater propensity to produce more massive remnants. There is some observational support for this hypothesis, in that there is some evidence that higher mass CSPN tend to have higher He/H ratios. However, the data (and the theory) are sufficiently uncertain to make all authors rather tentative. The existence of Sirius $\mathrm{B}$ as a massive (1.06 solar masses) DA white dwarf is a conspicuous counterexample to any putative trend, if a trend exists.

Nature of the Ejecta from the Superwind Phase: The simple existence of planetary nebulae requires that an evolutionary phase characterized by fast mass loss rates (> roughly $10^{-6}$ solar masses/yr) must exist (e.g., Renzini 1983). Observations of OH/IR stars confirm that a "superwind" phase exists, with slow velocities $(15 \mathrm{~km} / \mathrm{sec})$ and with mass loss rates of $10^{-5}$ to $10^{-4}$ solar masses/yr. Following this phase, CSPN continue to lose mass, with higher velocities (2000 $\mathrm{km} / \mathrm{s})$ but lower mass loss rates $\left(10^{-10}\right.$ to $10^{-7}$ solar masses per year; see, e.t., 
Habing 1988). We can see the nature of the matter which is pushed off the star in these evolutionary phases by observing and analyzing the most spectacular manifestation of the planetary nebula phenomenon, namely the nebula itself. Two important objects which may be telling us something important about the origins of the DA and non-DA white dwarfs are the planetary nebulae Abell 30 and Abell 78, objects sufficiently unusual that Mendez has put them in a class by themselves in classifying CSPN (Mendez et al. 1986). The nebulae in these objects contain regions which are almost pure He (Hazard et al. 1980, Jacoby and Ford 1983). I find that this is clear evidence that in some cases at least the superwind phase has reached down below the hydrogen envelope and laid bare the He layer, surrounding a degenerate $\mathrm{C} / \mathrm{O}$ core.

As is the case with photospheric compositions, the extreme cases of Abell 30 and Abell 78 represent the bitter end of a distribution of nebular chemical compositions which shows, in many cases, evidence that the mass loss process has reached down into stellar layers which have been affected by nucleosynthetic processes. Peimbert and Torres-Peimbert (1983) review work in which they have identified a class of nebulae enriched in He and N. C enrichments are also observed in many objects. Correlations between these enrichments and various stellar evolutionary hypotheses tend to fit general trends in both theory and observation, though details remain to be worked out (Kaler 1985). While the we are a long way away from a clear model which can be tested definitively, there is a clear indication that in many cases the mass loss process which learls to the formation of planetary nebulae digs down to the He layer of the AGB star and exposes processed material.

Generalities and Sugcestions for Future Work: Whether or not there is a direct connection between the $\mathrm{H}$-deficient CSPN and/or the H-deficient planetary nebulae and the non-DA white dwarfs, clearly stars which are about to become white dwarfs can scarcely be regarded as a homogeneous class of objects which are then divided into DA's and non-DA's by processes uccuring within the white dwarf phase of stellar evolution. The phenomenology strongly suggests a causal connection. A number of future lines of research are suggested on the basis of the above discussion:

Continued study of the composition of the central stars: Many of the conclusions cited above depend on the analysis of the spectra of CSPN by Mendez et al. (1988). The numbers are small, and it's not clear that the sample is not contaminated by selection effects which lead to the preferential discovery of nebulae of, for example, high surface brightness -. which may be related in some unknown way to various properties of the central star. Unfortunately the planetary nebula field has no equivalent of the PG survey, and selection effects may enter in unknown ways. Perhaps the only hope is to continue to study abundances of progressively fainter stars and to hope that selection effects, should they exist, will either become obvious, or that the sample will become 
sufficiently well-characterized that they become reduced to an acceptable level.

Particular open questions that suggest themselves are the following:

* What is the range of $\mathrm{He} / \mathrm{H}$ in the central stars?

* Is there any correlation between $\mathrm{He} / \mathrm{H}$ and other elemental abundances (particularly the abundance of the CNO elements)?

* How do central star abundance anomalies correlate with nebular abundance anomalies?

* What is the frequency of H-defecient central stars?

* Is there any correlation between H-deficiency and and $\mathrm{T}$ (eff) or the mass of the central star?

* What is the deathrate of the classical H-deficient stars?

Winds in Central Stars of Planetary Nebulae: IUE observations in particular have demonstrated that the very high luminosity planetary nebulae, stars with luminosities exceeding 500-1000 solar luminosities, are losing matter continuously as shown by the existence of P Cygni line profiles. For reviews see Heap (1983) and Perinotto (1983). While the wind velocities are high (up to $4000 \mathrm{~km} \mathrm{~s}^{-1}$ in the case of Abell 30 and 78; Kaler, Mo, and Pottasch 1985) the inferred mass loss rates are not too high and rather uncertain. Adopted rates range from $10^{-10}$ to $10^{-7}$ solar masses per year, though estimates exist for only a handful of stars. Over a planetary nebula lifetime of order $10^{3} \mathrm{yr}$, the most rapid mass loss rates could have a significant effect on the structure of the central stars. The $H$ layers left over from $A G B$ evolution contain about $10^{-4}$ solar masses of material; central stars losing mass at the highest rates which were quoted could completely deplete such a layer during the planetary nebula phase and greatly change the photospheric composition of the central star. It is an open question as to whether this occurs in any star, not to mention whether the phenomenon is sufficiently widespread as to be interesting in an overall evolutionary context. Winds in CSPN are a sufficiently new phenomenon that there are many obvious questions which still need answers. Mass loss rates need to be placed on a firmer footing; the types of stars which do have winds need to be delineated more precisely; and the duration of the wind phase needs to be estimated.

The PGI159 Stars: An additional puzzle regarding the nature of pre-white dwarfs is suggested by the existence of the PGil159 stars, which, considererl together with the unusual object H1504+65, occupy the same region of the HR diagram which contains the CSPN. The space density of the PGI159 stars derived by Green and Liebert (1987) of $3 \times 10^{-8} \mathrm{pc}^{-3}$, combined with a lifetime of $10^{5} \mathrm{yr}$ suggested by empirical determinations of $\mathrm{dP} / \mathrm{dt}$ (Winget et al. 1985), suggest.s a birthrate of $3 \times 10^{-14} \mathrm{pc}^{-3} \mathrm{yr}^{-1}$, about 0.1 times the birthrate of single white dwarf stars according to the statistios of Fleming, Liebert, and Green (1986). A more precise delineation of the duration of the "PGl159" phase will be required ts 
determine whether $10^{6} \mathrm{yr}$ is really the correct time to use in this calculation, since it refers to the e-folding time for a structural change in a PG1159 star rather than the time it takes for the star to cool through the PG1159 phase. Considering that this birthrate could be a factor of 3 or even 10 too low, it's possible that the PG1159 stars represent a significant feeder channel into the white dwarf part of the HR diagram. A more precise determination of the space density and birthrate of PGl159 stars, which is in principle possible with better interpretation of their spectra and with discovery of additional objects from the southern hemisphere surveys, is another important open question.

My principal point in calling attention to the existence of the PG 1159 stars is that, considering them and the CSPN together (which one certainly should since they occupy the same part of the HR diagram), there are a number of different types of stars feeding into the upper end of the white dwarf cooling sequence. We don't know the $\mathrm{He} / \mathrm{H}$ ratios of the $\mathrm{PG}$ stars precisely but suspect they are certainly $>1$ (Wesemael, Green, and Liebert 1985), corresponding to $y>0.5$. This strengthens the conclusion that white dwarf precursors have a wide range of $\mathrm{He} / \mathrm{H}$ ratios. The analysis of $O$ subdwarfs by Kudritzki and collaborators (see Kudritzki 1988; see also Thejll, Charache, and Shipman, in this volume) indicate a similar range of He abundances.

Additional Evidence for Particular Primordial Scenarios: The evidence discussed above regarding the nature of the CSPN tends to support primordial models in general, entirely apart from the particular mechanism which is invoked to explain the origin of DA's and non-nA's. The particular scenario proposed most recently is the idea developed by Iben, Renzini, and co-workers that the phasing of the onset of the superwind, relative to the cycle of He shell flashes, determines whether a star will evolve into a DA or a non-DA. This particular scenario has the advantage of predicting roughly the right relative abundance of DA's and non-DA's (the predicted ratio is about $4: 1$, which broadly speaking reflects the actual distribution). In addition, the differences between the parent masses and total ages (not just cooling times) of the two types of white dwarfs are expected to be subtle at most. (Subtle differences might be important in explaining the changing ratio of DA:non-DA as a function of $T(a f f)$; see section IV below). While we can't observe parent masses and ages of white dwarfs directly, we can observe remnant masses and kinematic properties, which are correlated with total ages and parent masses. The differences between DA's and non-DA's are, at the present time, undetectable.

TII. MIXING SCENARIOS

One reason that letate about the origin of $\mathrm{DA}$ 's and non-DA's has become refreshingly active has been a proposal, articulated in increasing detail in 
recent years, that during the white dwarf cooling sequence DA's change into nonDA's and vice versa. The initial presentation of this idea was at the 6th European Workshop on White Dwarf Stars (Liebert, Fontaine, and Wesemael 1987); for subsequent articulations of it see Liebert 1986; Fontaine and Wesemael 1987: Vennes, Pelletier, Fontaine, and Wesemael 1988). Because of these recent reviews of the "case for thin layers," my discussion will be brief and will tend to distinguish from evidence which argues for mixing and accretion in general as opposed to evidence for this proposal that essentially all white dwarf stars have hydrogen layers of $10^{-13}$ solar masses.

In general, I define "mixing" scenarios as ones in which processes which transform non-DA stars into DA stars, or vice versa, account for the simultaneous: existence of both classes of objects. I refer to these as mixing scenarios since convective mixing, in contrast to accretion, seems to be the predominant process, especially so in the thin layer scenario. There are reasons (section $V$ below) to suspect that the role of accretion in the DA/non-DA bifurcation is minimal.

Number Statistics of DA and Non-DA Stars: The single most powerful argument in favor of the role of mixing processes in the chemical evolution of white dwarf stars is that the number ratio of DA's to non-DA's changes dramatically with effective temperature (Liebert 1986 and references therein). Figure 2 below, similar to a diagram in Liebert's review, summarizes the situation:

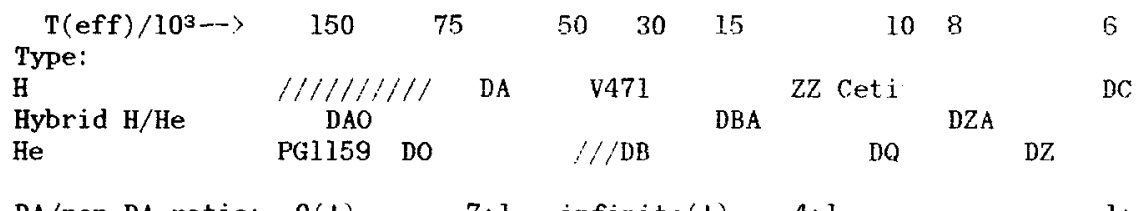

Figure 2: The major spectroscopic classes of white dwarf stars, and the ratio of the two major types, as a function of $\mathrm{T}$ (eff). Diagonal slashes $(/ / 1)$ represent regions of $\mathrm{T}$ (eff) where examples of that particular compositional class don' $t$ exist.

Several major transitions are indicated in Figure 2. Starting at the high temperature end of the white dwarf sequence, it is now well established (see, e.g., Fleming, Liebert, and Green 1986, Holberg 1987) that the hottest DA stars have temperatures of $80,000 \mathrm{~K}$ and that the hottest non-DA stars have considerably higher temperatures, with the extreme being $\mathrm{H} 1504+65$ with $\mathrm{T}($ eff $)=160,000 \mathrm{~K}$. At about $T$ (eff) $=45,000 \mathrm{~K}$, the non-DA stars disappear from sight for at least from the PG survey), to reappear at $\mathrm{T}$ (eff) $=30,000 \mathrm{~K}$. From $30,000 \mathrm{~K}$ to $12,000 \mathrm{~K}$, the DA/non-DA number ratio assumes its "canonical" value of 4:1. At very cool temperatures, the ratio increases to $1: 1$.

Proponents of the mixing scenario interpret these changing number ratios as evidence that DA's are changing into non-DA's and vice versa. The specific 
mechanism, as outlined by Fontaine and Wesemael (1987), is based on the proposition that the hydrogen layers in the DA stars are quite thin, 10-13 solar masses according to the latest numbers of Vennes et al. (1988). The scenario then works something like the following, as sketched by Fontaine and Wesemael (1987): At very high temperatures, all white dwarf stars appear as PG 1159 stars. The hydrogen is uniformly mixed through their outer layers and so these objects have He-dominated atmospheres, as observed. By the time a star cools to a temperature of $80,000 \mathrm{~K}$, hydrogen diffusing upward through the atmosphere makes some white dwarfs begin to appear as DA stars. By the time white dwarfs cool to a temperature of $45,000 \mathrm{~K}$, diffusion will have separated the mixtures in all white dwarfs, so that all white dwarfs appear as DA stars (explaining the "gap" in the $\mathrm{DB}$ sequence). At the lower end of the gap, at $\mathrm{T}$ (eff) $=30,000 \mathrm{~K}$, the helium convection zone will break through the thin $H$ surface layer, and transform those stars with thin $\mathrm{H}$ surface layers into DB stars again. Several billion years later, when temperatures between 5,000 and $10,000 \mathrm{~K}$ are reached, a deep convection zone in the DA's will turn most of the stars into non-DA stars, as was suggested many years ago (Strittmatter and Wickramasinghe 1971, Koester 1976, Vauclair and Reisse 1977, D'Antona and Mazzitelli 1979).

The Montreal group has pointed out that this scenario has the advantage of explaining the observed pattern of photospheric X-ray emission in DA white dwarf stars. Observations of white dwarfs in the $x$-ray spectral region with the EINSTEIN satellite (Petre, Shipman, and Canizares 1986) have suggested that some opacity source, widely interpreted as helium, increases in its relative abundance with increasing $\mathrm{T}$ (eff) in the DA stars. Subsequent observations with EXOSAT (Jordan et al. 1987, Paerels and Heise 1988, ApJ submitted) have clouded the picture somewhat; my own reading of the data is that the correlation is still there, though there are stars which do not appear to follow the abundance pattern (as the original white dwarf $\mathrm{X}$-ray source, $\mathrm{HZ} 43$, stands out as a conspicuous exception to the correlation). Vennes et al. (1988) contend that interpreting the X-ray absorber as $\mathrm{He}$, and interpreting the correlation as due to radiation pressure, requires the existence of very thin $H$ layers on the surfaces of hot DA white dwarfs. It is this analysis which produces the figure of $10^{-13}$ solar masses which is referred to so frequently in this paper. Another suggestion that the H layer mass is thinner than $10^{-4}$ solar masses comes from the interpretation of $\mathrm{ZZ}$ Ceti pulsations, discussed extensively in other papers at this conference (Brassard, Wesemael, and Fontaine, this volume).

Since the Montreal group is actively exploring this particular scenario, I won't suggest any open questions here. It is true that, at the moment, the only aspect of it where published papers explore it in quantitative detail is the use of thin layers and stratified atmospheres to explain the behavior of X-ray emission from DA white dwarfs. The major problem, as will be discussed in some detail below, is to find whether a consistent definition of what constitutes a 
"thin layer" can account for each of the major transitions outlined above, in a way that is consistent with the observations.

\section{DIFFICULTIES WITH EACH SCENARIO}

Each of the scenarios sketched above faces some solid pieces of evidence which must be reconciled with it. The changing number statistics illustrated in Fig. 2 present a serious challenge to someone who finds the simplicity of the primordial scenarios to be quite appealing. How can one explain the changing abundances of DA's and non-DA's if this fundamental characteristic is, like sex in human beings, set at the birth of a star as a white dwarf? Advocates of the mixing scenario must face, in general terms, the fact that the separation between He-rich and He-poor stars seems to be established prior to the entry of stars onto the white dwarf cooling sequence. In addition, the thickness of the $\mathrm{H}$ layer left after AGB evolution is sufficiently large that general evolutionary considerations would suggest "DA or non-DA forever:" Either the entire H layer is stripped off and the star remains as a non-DA, or that most of it remains $\left(\sim 10^{-4}\right.$ solar masses $)$ and it is forever a DA.

Primordial Scenarios: Here the principal problem appears to be the changing number ratios illustrated in Figure 2; some changes can be explained readily, and some cannot. The absence of DA's at $\mathrm{T}$ (eff) > 80,000 $\mathrm{K}$ seems to be one of the more tractable problems. The extension of $\mathrm{H}$ shell burning just prior to entry into the white dwarf cooling track can prevent a pre-white dwarf with a significant layer of $\mathrm{H}$ from contracting too much and becoming too hot. If there were more hot DA stars discovered (as their will be when the southern hemisphere surveys for faint blue stars, and the necessary follow-up spectroscopy, have been completed), the high temperature boundary of the DA stars can be established more precisely. It also may be possible that additional calculations of the evolution of hot prewhite dwarfs with hydrogen layers of various thicknesses would enlighten matters.

Can the primordial scenario explain the DA X-ray data? The Montreal group has shown that the naive interpretation of the data .... that radiation pressure pushes He up to the surface -- won't work. An interesting question for the future, which EUV spectroscopy can illuminate, is whether the $\mathrm{X}$ ray opacity source is indeed He. The few spectra which do exist don't show any promise (so far) of resolving this question unambiguously. When the Extreme Ultraviolet Explorer provides additional spectra in the early 1990s, we may be able to make significant progress.

The existence of the DB gap between 30,000 tand $45,000 \mathrm{~K}$ may prove to be one of the most difficult problems to solve, in the context of the primordial scenario. While there are some uncertainties in the no temperature scale, a sufficient number of different models were used by liebert et al. (1986) that it 
would seem difficult to change the temperature of the hottest DB (or the coolest Do) by 30\%, especially considering that model uncertainties generally affect lines more than continua, and the continua were used to establish the existence of the gap. Is it possible that DB's cool much faster than DA's in this temperature range? Differential cooling rates which were sufficiently large could solve the problem.

At the cool end, it seems reasonably clear that mixing does occur, as has been known for some time (see D'Antona and Mazzitelli 1979). As D'Antona (1986) emphasized, one can directly use the thicknesses of $\mathrm{H}$ convection zones established by various modelers in the 1970 s to determine the range of $H$ convective zone thicknesses. Greenstein's (1986) data show that the relative abundance of DA stars seems to start dropping by $9,000 \mathrm{~K}$ (though the numbers are small) and is definitely established by 7,000 K. The models published in D'Antona and Mazzitelli indicate that for at least some DA stars the $H$ shell has thinned out to $10^{-9}$ solar masses. The persistence of DA compositions at very low temperatures (around $5,000 \mathrm{~K}$ ) indicate that some DA stars have $\mathrm{H}$ layers as thick as roughly $10^{-6}$ solar masses.

Because most expectations are that the hydrogen shells left on red giant stars will have masses of 10-4 solar masses, Greenstein's data, interpreted at face value, may present a real problem to the primordial scenario. While there are difficulties in observing the very coolest stars, and even more serious difficulties around the interpretation of the observations, the diminution of the $\mathrm{DA} /$ non-DA value from its canonical value of $4: 1$ is clearly established at temperatures $(>7,000 \mathrm{~K})$ where the interpretation of the spectra is reasonably secure. One way around this problem is diffusion induced hydrogen burning (Michaud, Fontaine, and Charland 1984), though Iben and MacDonald (1985, 1986) suggest that only a factor of 2 reduction in envelope thickness can be obtained. Other possibilities are that winds in CSPN, or possible in the white dwarf phase itself (such as the shortward-shifted features detected by Bruhweiler in ILE observations) may play a role. Still another possibility is that there is some physics missing (convective overshoot? rotation? convection in partially degenerate layers?) in the models of the envelopes of cool DA stars which produces mixing with layers of $10^{-4}$ solar masses, though D'Antona (1986) believes that the stability of such thick layers seems well determined. Another possibility (Iben and MacDonald 1985,1986 ) is that the very coolest white dwarfs are the descendants of a different stellar population than the hotter ones, and that characteristics of this population are more favorable for the formation of non-DA stars.

Outstanding problems suggested by the foregoing discussion are:

* What is the temperature of the hottest DA star?

* Is He opacity the real interpretation of the X-ray observations of hot DA 
* Is there any way of explaining the "DB gap"?

* Just when does the DA/non-DA ratio change from $4: 1$ to $1: 1$ ? (This will require better model atmospheres as well as additional observations.)

* Can diffusion induced hydrogen burning work?

* Do CSPN winds affect the thickness of the H layer in DA stars?

* Is there any way to $\mathrm{mix} 10^{-4}$ solar masses of hydrogen into the interiors of cool DA stars?

Mixing Scenarios: These scenarios also face a number of difficulties. Some of the difficulties are particular to the thin-layer proposal recently discussed by the Montreal group, but some seem endemic to any scenario which appeals mainly to processes occurring within the white dwarf evolutionary stage to account for the existence of DA's and non--DA's. The two most fundamental difficulties in the latter category have been recognized for some time:

How does $10^{-4}$ solar masses of $H$ thin out to $10^{-13}$ solar masses during the planetary nebula ejection stage? It is true that even in the primordial scenarios also, apparently, require a reduction in the thickness of the hydrogen layer in order to produce mixing at cool temperatures. However, in the primordial scenario, there is $10^{9}$ years of white dwarf evolution to do the job. In addition, diffusion will tend to concentrate $H$ layers at the surface of the star, so if whatever is destroying the $\mathrm{H}$ operates at the interior of the star, there is a natural mechanism which can thin out the $H$ laver and still leave a little remaining at the surface.

The mixing scenario requires a much shorter time scale for thinning out the $H$ layer. If one interprets the scenario in its simple form, where the ancestors of all white dwarf stars are "PG1 159" stars, then only $10^{2}$ to $10^{4}$ years separates a DA star's departure from the AGB from its entry onto the white dwarf cooling sequence where, apparently, all stars have 11 layer masses very close to $10^{-13}$ solar masses. To be fair, however, the observations only require that the thin layer be estabilshed by temperatures of order $45,000 \mathrm{~K}$, giving one a (little) more time. There is the difficulty that to account for the absence of $D B$ stars, the $X$ ray observations, and the presence of $\mathrm{DB}$ stars with $\mathrm{T}(\mathrm{eff})$ just below $30,000 \mathrm{~K}$, virtually all white dwarfs have a thin $\mathrm{H}$ layer on the top of precisely the same thickness. This thickness is of order 1 part of $10^{9}$ of the original H layer. It is hard to imagine a mechanism for thinning the $\mathrm{H}$ layer which is this finely tuned, which will always remove all but $10^{-9}$ of the $H$ (and not leave more or remove it completely?

Low can the mixing scenario be reconciled with the range of He/H ratios found in white dwarf precursors? Characteristics of white dwarf precursors suggest strongly, though do not compel, that the distinction between IDA and non-DA stars has been estahlished among the central stars of planetary nebulae (see above). As 
the mixing scenario has been presented so far, the PG 1159 stars have been seen as the ancestors of all white dwarfs. This is incoriect. The temperatures and the birthrates of PG1159 stars indicate that they evolve in parallel with the planetary nebula central stars, not as their evolutionary descendants. The deathrates of PG1 159 stars are only $1 / 10$ of the birthrate of white dwarfs, but could be as high as the birthrate of non-DA white dwarfs (which is $1 / 4$ the birthrate of DA white dwarfs in the primordial scenario, and agrees within uncertainties with the deathrates of PGl159 stars). Within the uncertainties, it is easily possible that all non-DA white dwarfs are made from PGl159's. The role of the sdo stars is yet to be determined.

In any case, if the mixing scenario is to remain viable, it has to account for a variety of compositions among white dwarf precursors.

The next indication of compositional change is at the red end of the IB gap, where stars are purported to change from all DA's to a mixture of DA's and DB's. Some of the hottest $\mathrm{DB}$ stars are the DB variables, just below this gap, with only PG $0112+104$ being a nonvariable DB hotter than the DB instability strip. Small aperture IUE observations currently under way can demonstrate or contradict the presence of $\mathbf{H}$ in the hottest $\mathrm{BB}$ 's just below the gap (Shipman and Liebert, in preparation). D'Antona and Mazzitelli's (1979) graphs indicate that the thickness of the He convective layer is rather small at $\mathrm{T}$ (eff) $=30,000 \mathrm{~K}$, suggesting that the residual $\mathrm{H}$ might still be visible. Further theoretical exploration of the expected $\mathrm{H}$ abundance in the hottest $\mathrm{DB}$ stars would be desirable.

While the thin-layer scenario explains the differing ratios of DA's to non DA's illustrated in Figure 2, much work remains to be done until (and if) it is to be accepted as an explanation for the chemical evolution of white dwarf stars. Part of the job, which the Montreal group is actively working on, is a theoretical investigation of envelope evolution in an effort to determine whether the scenario sketched out in the previous paragraph does indeed work. In particular, are the envelope thicknesses envisaged for particular types of white dwarfs consistent with the whole picture? For example, if you postulate that hot DA white dwarfs have a hydrogen layer of $10^{-13}$ solar masses, can you still explain, on a quantitative basis, why the next mixing phenomenon starts at $10,000 \mathrm{~K}$ and is not complete until 6,000 K? D'Antona (1986, referring to earlier work by D'Antona and Mazzitelli 1979) suggests that such a thin layer would mix at hotter temperatures. The following questions occur to me as ones which may be particularly ripe for observational or theoretical investigation at the present time:

* When convective mixing turns DA stars into non-DA stars at $30,000 \mathrm{~K}$ is the transition complete and abrupt, or is there a temperature range at which we expect to find a substantial number of stars with mixed composition? Is this where stars like GD 323 (Liebert et al. 1984) fit in? Would one expect all DB stars just below the $30,000 \mathrm{~K}$ transition to have at least some residual $\mathrm{H}$ ? 
* How can this scenario account for the existence of at least some DA stars at temperatures considerably cooler than $10,000 \mathrm{~K}$ (Greenstein 1986)?

* Is it really He that is providing the $X$-ray opacity in the hot DA stars?

* Green and Liebert (1987) argue that the number densities of PG 1159 and DO stars are inconsistent with these objects serving as the only feeder channel into the white dwarf cooling sequence. Is it possible to incorporate the CSPN as a separate feeder channel into this scenario?

\section{A MODEST PROPOSAL}

With all of the difficulties presented by each scenario, let me close by suggesting a modest proposal, largely unoriginal, which seems to be least inconsistent with the observational data. While it is largely similar to the primordial scenario proposed above, there is an important variation on the theme which is subject to experimental test. The proposed scenario is presented below in Figure 3.

$$
\text { H layer mass } \quad 10^{-4} \quad 10^{-7} \text { to } 10^{-9}
$$

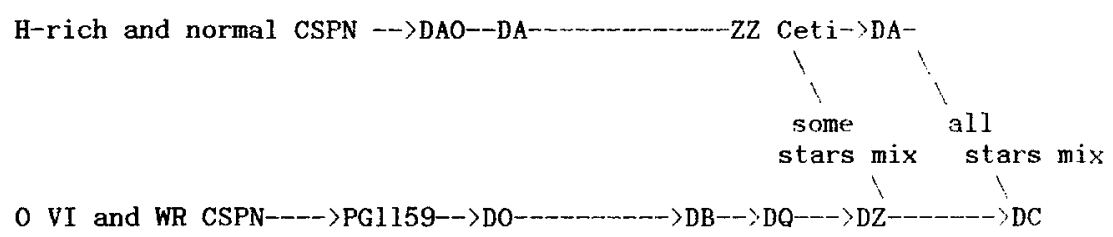

He layer mass $\quad 10^{-2} ? \quad 10^{-4}$

Figure 3. A modest proposal for the chemical evolution of white dwarf stars.

This proposal is basically a two-channel scenario with some additional provisos. In the DA channel, some process -- mass loss and diffusion induced nuclear burning are two possibilities - reduces the H layer mass from its initial value of $10^{-4}$ solar masses, the most plausible value from the late stages of red giant evolution, to the value of $\left\langle 10^{-7}\right.$ which seems to be now required by our interpretation of the $\mathrm{ZZ}$ Ceti stars. A range of $\mathrm{H}$ layer masses is most consistent with the statistics of very cool DA stars, allowing some DA stars to turn into non-DA stars by convective mixing at temperatures like 9,000 $\mathrm{K}$, with others persisting as DA's down to around $5,000 \mathrm{~K}$.

The non-DA channel is basically similar to that outlined in the primordial scenario, but I mention one additional circumstance. The explanation of the DO stars as resulting from convective dredge-up of the carbon by Fontaine et al. (1984) suggests, on the basis of rather preliminary calculations, that the thickness of the He layer required to mix the DQ stars should be about $10^{-4}$ solar masses. While the calculations are rather rough and the agreement with the 
functional form of the ( $\mathrm{C} / \mathrm{He}$ ) versus $\mathrm{T}$ (eff) relation is a bit approximate, the conclusion that the He layer must be thinner than the canonical 10-2 solar masses left at the end of the red giant stage seems reasonably firm.

These considerations suggest that some mechanism operates in both channels to reduce the thickness of the outermost layer of white dwarf stars. It does not have to be as finely tuned as in the mixing scenarios, and it can operate over the $10^{9}$ years which separate a newly born white dwarf from the $10,000 \mathrm{~K}$ range where we empirically determine white dwarf envelope thicknesses. A modest stellar wind is one possibility; the required mass loss rates are 10-11 solar masses/yr for the non DA's and 10-13 solar masses/yr for the DA's. Detection of such a wind is remotely possible for the $\mathrm{DA}^{\prime} \mathrm{s}$, depending on its temperature and velocity structure. If diffusion induced nuclear burning can be made to work, this is another way of thinning down the $\mathrm{H}$ layer. For this proposal to be viable, of course, we must find a way around the phenomena which it can't explain: the $X$-ray emission from DA stars and the DB gap are the most serious (see section IV above).

Little has been said about accretion in this paper. Two recently discovered pieces of evidence suggest that accretion from the ISM has little to do with whether a particular white dwarf becomes a DA or a non-DA star. Oswalt et al. (1988) discovered a binary system containing a DA star and a DB star. Since these stars have shared a common trajectory through the interstellar medium (and thus a common accretion history), accretion has had nothing to do with the DA/DB bifurcation in this case. In addition, recent investigations of several $\mathrm{DBAZ}$ stars (Kenyon, Shipman, Sion, and Aannestad 1988, Kenyon, Sion, and Aannestad 1988) suggest that the $H$ in these stars comes from accretion, in other words that non-DA stars can successfully resist $H$ pollution.

I thank Alvio Renzini for his cogent and well-deserved criticism of my talk at Mexico City which led to a better understanding on my part of how important the diversity among planetary nebula central stars is in the context of the problem discussed here, and I thank my colleague Jim MacDonald for discussions. I also thank the National Science Foundation (grant AST 87-20530) and NASA (NAG 5-972 and NAG 5-348) for financial support.

REFERENCES

D'Antona, F., and Mazzitelli, I. 1979, Astr. and Ap., 74, 161.

D'Antona, 1986, Memorie della Societa Astronomica Italiana 58, 123.

Fleming, T.A., Liebert, J., and Green, R.F. 1986, Ap.J. 308, 176.

Fontaine, G., Villeneuve, B., Wesemael, F., and Wegner, G. 1984, Astrophys.J. (Letters) 277, L61.

Fontaine, G., and Wesemael, F, 1987 Proceedings of IAU Colloguium 95: the Second 
Conference on Faint Blue Stars, D.S. Hayes, I. Liebert, and A.G.D. Philip, eds., (Schenectady: L. Davis Press), 319.

Green, R.F. and Liebert, J. 1987, IAU Colloquium 95, 261.

Greenstein, J.I. 1986, Astrophys..J., 304, 334.

Habing, H., 1988, in Torres-Peimbert 1988, in press.

Hazard, C. et al. 1980, Nature 285, 463.

Heap, S.R. 1983, in IAU Symposium 103: Planetary Nebulae, ed. D.R. Flower, (Dordrecht: Reidel), 375.

Holberg, J. 1987, IAU Colloquium 95, (see: Fontaine and Wesemael above), 285.

Iben, I., jr. 1984, Ap.J. 277, 333.

Iben, I., jr, and MacDonald, J. 1985, Ap. J., 296, 540.

Iben, I., jr, and MacDonald, J. 1986, Ap. J., 301, 164.

Iben, I., jr., and Renzini, A. 1983, Ann. Rev. Astr. Ap. 21, 271.

Jacoby, G. H., and Ford, H.C. 1983, Astrophys. J. 266, 298.

Jordan, S., Koester, D., Wulf-Mathies, C., and Brunner, H. 1987, Astron. Astrophys., in press.

Kaler, J.B., Mo,J.H., and Pottasch, S.R. 1985. Astrophys.J. 288, 305.

Kaler, J.B. 1985, Ann. Rev. Astron. Astrophys. 23, 89.

Kenyon, S., Shipman, H., Sion, E., and Aannestad, P. 1988, Astrophys. I. Letters, (in press).

Kenyon, S., Sion, F., and Aannestad, P. 1988, Astrophys. J. Letters, in press. Koester, D. 1976, Astron. Astrophys. 52, 415.

Kudritzki 1988, in Torres-Peimbert 1388.

Liebert, J. 1986, in Proceedings of IAU Colloguium No. 87: Hydrogen-Deficient Stars and Related Objects, eds. K. Hunger, D. Schonberner, and N. K. Rao, (Dordrecht: Reidel), p. 367.

Liebert, J., Wesemae1, F., Sion, E.M., and Wegner, G. 1984, Ap.J., 277, 692.

Liebert, J., F. Wesemael, C. Hansen, G. Fontaine, H. Shipman, E. Sion, D. Winget, and R. Green, Ap.J. 309, 241.

Liebert, J., Fontaine, G., and Wesemael, F. 1987, in Memoria della Societa Astronomica Italiana: 58, 17.

Mendez, R.H., Miguel, C.H., Heber, U., and Kudritzki, R.P. 1986, in Proceedings of IAU Colloquium No. 87: Hydrogen-Deficient Stars and Related Objects, eds. K. Hunger, D. Schonberner, and N. K. Rao, (Dordrecht: Reidel), p. 323.

Mendez, R.H. 1987, IAU Colloquium 95 (see Fontaine and Wesemael above), 191.

Mendez, R.H., Kudritzki, R.P., Herrero, A., Husfeld, D., and Groth, H.G. 1988, Astron. Astrophys. 190, 113.

Mendez 1988, in Torres-Peimbert 1988, in press.

Michaud, G., Fontaine, G., and Charland, Y. 1984, Ap.J., 280, 247.

Oswalt, T., Sion, E., and Hintzen, P. 1988, Astrophys.J. Letters, in press.

Paerels, F., and Heise, J. 1988, Astrophys. J., submitted.

Peimbert, M., and Torres-Peimbert, S. 1983, in IAU Symposium 103:Planetary 
Nebulae, ed. D.R. Flower, (Dordrecht: Reidel), 375.

Perinotto, M. 1983, IAU Sumposium 103.

Petre, R., Shipman, H.L., and Canizares, C.L. 1986, Ap.J. 304, 356.

Renzini, A. 1988, in Torres-Peimbert 1988.

Renzini, A. 1983, in Planetary Nebulae, ed. D. R. Flower, p. 267.

Schatzman, E. 1958, white Dwarfs.

Schonberner, D. 1977, Astr. Ap. 57, 437.

Shipman, H. 1988, in Torres-Peimbert 1988.

Shipman, H. 1987, IAU Colloquium 95, (see Fontaine and Wesemael above), 273.

Shipman, H.L. 1972, Ap. J.,177, 723.

Sion, E.M. 1986, Publ. Astrun. Soc.Pac. 98, 821, 1986.

Sion, E. M., Liebert, J., and Starrfield, S.G. 1985, Astrophys.J. 292, 471.

Strittmatter, P.A., and Wickramasinghe, D.T. 1971, Mon. Not. Foy. Astron. Soc., $152,47$.

Torres-Peimbert, s., ed. 1988 Proceedings of IAI Symposiun 131: Planetary Nebulae, in press.

Vauclair, G., and Reisse, C. 1977, Astron. Astrophys. 61, 415.

Vennes,S., Pelletier, C., Fontaine, G., and Wesemael, F. 1988, Astrophys.J. (in press).

Wesemael, F., Green, R.F., and Liebert, J. 1985, Ap. J. Suppl., 58, 379.

Winget, D., Kepler, S., Robinson, E.L., Nather, K.E., and O'Donoghue, D. 1985, Ap.J. 292, 606. 